Service social

\title{
L'évaluation régionale de la politique de la santé et du bien-être. Quand une nouvelle perspective s'impose
}

\section{Sylvie Cantin et Jean Beaudry}

Volume 47, numéro 1-2, 1998

Évaluation - Colloque 1999

URI : https://id.erudit.org/iderudit/706786ar

DOI : https://doi.org/10.7202/706786ar

Aller au sommaire du numéro

Éditeur(s)

École de service social de l'Université Laval

ISSN

1708-1734 (numérique)

Découvrir la revue

Citer cet article

Cantin, S. \& Beaudry, J. (1998). L'évaluation régionale de la politique de la santé et du bien-être. Quand une nouvelle perspective s'impose. Service social, 47(1-2), 153-170. https://doi.org/10.7202/706786ar
Résumé de l'article

Cinq ans après le dévoilement de la Politique de la santé et du bien-être (PSBE), quel était le chemin parcouru? Quels étaient les ajustements à faire? Voilà les questions de départ de notre évaluation. Les approches habituellement employées pour l'évaluation de politiques ne s'avérant d'aucun secours pour y répondre, il fallait trouver une méthode de remplacement. Il a donc été nécessaire de faire preuve de créativité pour réaliser une étude qui soit rigoureuse tout en permettant aux décideurs d'ajuster le tir avant la date d'expiration du délai pour réaliser les objectifs, soit 2002. Le présent article fait le point sur les considérations méthodologiques relatives à la démarche élaborée pour l'évaluation formative de la PSBE en Montérégie. 


\title{
L'évaluation régionale de la Politique de la santé et du bien-être Quand une nouvelle perspective s'impose ${ }^{1}$
}

\author{
Sylvie CANTIN \\ Jean BEAUDRY
}

Cinq ans après le dévoilement de la Politique de la santé et du bien-être (PSBE), quel était le chemin parcouru? Quels étaient les ajustements à faire? Voilà les questions de départ de notre évaluation. Les approches habituellement employées pour l'évaluation de politiques ne s'avérant d'aucun secours pour y répondre, il fallait trouver une méthode de remplacement. Il a donc été nécessaire de faire preuve de créativité pour réaliser une étude qui soit rigoureuse tout en permettant aux décideurs d'ajuster le tir avant la date d'expiration du délai pour réaliser les objectifs, soit 2002. Le présent article fait le point sur les considérations méthodologiques relatives à la démarche élaborée pour l'évaluation formative de la PSBE en Montérégie.

Five years after the appearance of the Policy on Health and Well-Being, what has been achieved? What adjustments are required? These were the preliminary questions our evaluation had to address. But traditional methods used to evaluate policies were of no help in answering them. Therefore, we had to be innovative as our study had to be both rigorous and easy to use by decision-makers who have to make adjustments before the deadline for the attainment of the objectives (i.e. 2002). This paper addresses the methodological issues related to the formative evaluation of the policy in Montérégie.

1. Le lecteur intéressé au rapport d'évaluation de la PSBE, en version intégrale ou abrégée, est invité à commander une copie auprès de $M^{\text {me }}$ Ginette Charbonneau de la Direction de la santé publique de la RRSSS de la Montérégie. 


\section{Problématique}

La Politique de la santé et du bien-être (PSBE) constitue un véritable guide quant aux voies à privilégier dans la lutte aux problèmes de santé et aux problèmes sociaux qui affectent le plus la population. Ses lignes directrices interpellent les acteurs du domaine de la santé et ouvrent de nouvelles perspectives à la contribution de ceux des autres secteurs de la vie collective. La publication, en 1997, des Priorités nationales de santé publique : 1997-2002 vient en réitérer l'actualité, car celles-ci s'inscrivent dans la perspective des objectifs de la PSBE et visent à contribuer à leur atteinte.

Le 30 mars 1995, le conseil d'administration de la Régie régionale de la santé et des services sociaux de la Montérégie adoptait un Cadre de référence pour la distribution de nouvelles ressources financières destinées aux priorités régionales découlant de la Politique de la santé et du bien-être. Dans ce cadre de référence figuraient le mandat d'évaluation ainsi qu'une proposition d'allocation pour sa réalisation.

Quelques années après le dévoilement de la PSBE, la préoccupation à l'endroit des éventuels résultats de la politique était donc déjà palpable. Quel chemin avait été parcouru? Quels étaient les ajustements à faire? Ce sont là les premières questions sur lesquelles nous avons entrepris de nous pencher. Les avenues pouvant fournir des éléments de réponse s'avéraient cependant, à ce moment, plutôt limitées.

En effet, lorsqu'il s'agit de l'évaluation d'une politique et non d'un programme, lorsque cette politique s'intéresse sans hiérarchisation des priorités à dix-neuf objectifs de santé, lorsque son territoire d'implantation est scindé en sept sous-régions, lorsque les ressources humaines et financières sont limitées, il faut faire preuve de créativité pour réaliser une évaluation à la fois rigoureuse et informative. Le présent article vient donc faire le point sur les considérations méthodologiques relatives à la démarche élaborée pour l'évaluation de la PSBE en Montérégie.

\section{FINALITÉ POURSUIVIE}

Les écrits scientifiques dans le domaine de l'évaluation de programmes recèlent une diversité de buts et de justifications relatifs aux activités d'évaluation. Bien que l'objectif ultime de toute évaluation soit de 
fournir une rétroaction qui permette de porter un jugement critique sur les actions entreprises, le chemin emprunté pour y arriver n'est pas unique. Ainsi, Stecher et Davis (1987) recensent cinq avenues possibles, chacune offrant sa propre perspective sur la façon d'envisager l'évaluation et chacune exigeant de l'évaluateur un rôle quelque peu différent. Parmi celles-ci, deux insistent plus particulièrement sur les résultats du programme évalué, deux autres s'intéressent à l'utilisation éventuelle de l'information et enfin une dernière se centre sur la description du programme. Nous avons donc eu à prendre position.

Dans une perspective d'utilisation de l'information, une des avenues met l'accent sur l'aide à la prise de décision. Cette avenue nous est apparue tout indiquée : en effet, comme nous nous intéressions à l'état de la PSBE à mi-parcours, il allait de soi que l'évaluation que nous étions sur le point d'entreprendre devait fournir aux divers décideurs concernés des informations qui leur permettraient d'ajuster le tir avant la date d'expiration du délai pour l'atteinte des objectifs, soit 2002. Forts de cette position, nous avons donc eu des rencontres avec les utilisateurs potentiels des résultats de l'évaluation afin de déterminer avec eux la nature des informations requises.

\section{APPROCHES TRADITIONNELLES}

Une portion infime des écrits dans le domaine de l'évaluation s'intéresse à la question de l'évaluation de politiques, la grande majorité traitant de programmes, ou bien de projets. Un survol rapide des principales tendances au cours des vingt dernières années nous informe que deux approches sont généralement applicables lors de l'évaluation d'une politique.

La première approche cherche à en respecter l'intégrité en examinant de manière globale sa pertinence, ou encore son efficience ou son impact. C'est le cas par exemple des politiques québécoises à l'égard des personnes ayant des incapacités, où l'auteur s'est entre autres intéressé à l'analyse de la cohérence (Bolduc, 1986). C'est aussi le cas de la PSBE pour laquelle une stratégie actuellement fort répandue consiste à faire un suivi annuel des indicateurs élaborés par la Direction générale de la planification et de l'évaluation du ministère de la Santé et des Services sociaux (MSSS, 1995). 
La seconde approche consiste à examiner une politique par l'intermédiaire de certains des programmes à travers lesquels elle s'actualise. On s'en remet alors à une évaluation de programmes multiples dont les résultats servent d'indicateurs de performance de l'objet d'intérêt central. C'est d'ailleurs l'approche privilégiée par Béland (1983) dans l'évaluation de la politique des services à domicile du ministère des Affaires sociales et celle proposée dans la politique québécoise en matière de nutrition (ministère des Affaires sociales, 1977).

\section{L'approche globale}

L'approche, que nous qualifierons de " globale ", est apparue insatisfaisante, et ce, pour deux raisons. Premièrement, l'évaluation de la pertinence n'était plus appropriée puisque la PSBE était déjà en pleine implantation.

Quant à la mesure des effets, qui constitue pourtant la principale approche à laquelle recourir en pareil cas, elle posait un nombre élevé d'obstacles, dont certains étaient insurmontables. Son opérationalisation la plus courante, comme nous l'avons précédemment mentionné, consiste à recourir à un ensemble d'indicateurs qui nous auraient informés du degré d'atteinte des objectifs fixés. Toutefois, la mesure de l'atteinte des objectifs de la PSBE constitue une entreprise complexe, et ce, pour plusieurs raisons. D'une part, on remarque que les objectifs visés par la politique ne sont pas tous quantifiés. C'est le cas par exemple de l'objectif 16 qui vise d'ici l'an 2002 à « diminuer les problèmes de santé mentale ». Par ailleurs, il peut être difficile parfois d'obtenir une mesure de base à laquelle comparer les éventuels progrès. Par exemple, l'objectif 4, qui se lit : “ D'ici l'an 2002, diminuer les cas de violence faite aux femmes en milieu familial ». Dans cet objectif, non seulement on ignore de combien on vise à diminuer le problème, mais il est également difficile d'obtenir une mesure fiable du nombre de cas de violence faite aux femmes en milieu familial. L'imprécision de la mesure de base, de la quantité à y retrancher ainsi que de la mesure des progrès ne peut que mener à l'impossibilité de mesurer adéquatement le degré d'atteinte d'un objectif.

Enfin, certains objectifs ne sont tout simplement pas mesurables, comme l'objectif 5 : «D'ici l'an 2002, prévenir l'itinérance [...] 》 (l'italique est de nous). 
Par ailleurs, les données requises pour procéder au calcul d'indicateurs de santé et de bien-être pour une année donnée ne sont généralement disponibles qu'un an ou deux après leur collecte. Ainsi, pour le calcul d'indicateurs concernant l'année 1997, année où la PSBE était à mi-parcours, il aurait fallu attendre 1998, voire 1999, rien que pour disposer des données brutes!

De toute façon, il était vain de croire que cinq années de règne de la politique, surtout dans le contexte de restrictions budgétaires qui s'est rapidement imposé, auraient suffi pour produire des effets détectables à travers les indicateurs.

Enfin et surtout, pour qui se soucie de l'aspect formatif d'une évaluation, cette stratégie présente une faille conceptuelle importante. En effet, dans l'éventualité d'un écart substantiel entre les valeurs des indicateurs et les objectifs visés, que sait-on des rectificatifs à apporter, des ajustements à faire? En contrepartie, dans l'hypothèse où les résultats obtenus s'avèrent satisfaisants, dans quelle mesure peut-on postuler que ceux-ci sont tributaires de l'application de la politique? Bref, l'approche par indicateurs ne s'avérait, pour nous, d'aucun secours pour évaluer la PSBE à mi-parcours.

\section{L'approche par programmes}

En ce qui a trait à l'approche par programmes, son utilisation est davantage indiquée lorsque la politique à évaluer couvre un champ d'action relativement précis et restreint : de cette façon, il est facile de comparer les programmes entre eux. Cette méthode permet de circonscrire ceux qui donnent les résultats les plus probants ou, encore, qui s'avèrent les plus efficients. On comprendra facilement qu'avec dix-neuf objectifs de santé couvrant l'ensemble de la population et près de quatre-vingts voies d'action, la PSBE se prête difficilement à ce type d'approche. Qui plus est, le contexte extrêmement mouvant dans lequel s'amorçait l'évaluation suggérait de garder certaines distances par rapport à des activités ou programmes sur le terrain. En effet, leur survie était trop souvent imprévisible en raison des restrictions budgétaires qui sévissaient alors.

Par ailleurs, le contexte très particulier de la région d'investigation (Montérégie), où tous les objectifs de la PSBE ont été retenus comme prioritaires, aurait mené à la sélection d'un nombre considérable de 
programmes pour l'évaluation. Cette façon de faire aurait exigé les services d'une quantité de ressources humaines et financières dont ne dispose pas la Régie régionale.

Bref, l'approche par programmes se révélait tout aussi inapplicable que l'approche globale. II a donc fallu nous employer à élaborer une stratégie qui permette d'apporter des éléments d'information dynamiques à partir desquels on pourrait déceler si l'implantation de la PSBE est sur la bonne voie. De surcroît, il fallait tenir compte des besoins exprimés par les utilisateurs potentiels consultés.

\section{L’approche privilégiée}

Outre que l'approche retenue devait constituer une aide à la prise de décision, nous nous étions imposé d'élaborer une stratégie qui serait à l'abri de toute variable situationnelle pouvant menacer la réalisation de la démarche évaluative.

L'approche finalement adoptée consiste à envisager la Politique de la santé et du bien-être comme tout programme qui viserait, à partir d'une gamme de ressources et de moyens, l'atteinte de résultats clairement identifiés. Avec cette nouvelle assise, les modèles couramment utilisés dans le domaine de l'évaluation de programmes deviennent applicables à la PSBE.

Une telle approche demande cependant qu'on résolve deux difficultés majeures : 1) traduire les composantes de la PSBE en des éléments pouvant être soumis aux mêmes types de grilles d'analyse que celles créées à des fins d'évaluation de programmes et 2) élaborer une méthodologie qui assure la validité de la démarche. II nous a donc fallu effectuer des choix relativement à ces deux domaines.

\section{LES CHOIX RELATIFS AUX COMPOSANTES DE LA DÉMARCHE D'ÉVALUATION}

\section{Les objets d'évaluation}

La Politique de la santé et du bien-être s'inscrit dans le prolongement de la réforme du système de services. Elle définit la nouvelle orientation qui doit désormais guider l'organisation et le fonctionnement du réseau de la santé et des services sociaux. Mais, élément encore plus fondamental, si elle mobilise suffisamment et 
permet une diminution de la prévalence et de l'ampleur des principaux problèmes de santé, d'adaptation et d'intégration sociales, on peut émettre l'hypothèse suivante : la politique aura des retombées positives pour le développement économique et social du Québec (Politique de la santé et du bien-être, p. 185).

Comme nous sommes à même de le constater, la PSBE mise sur la mobilisation des acteurs et sur les répercussions des actions en promotion/prévention pour atteindre son but ultime : améliorer la santé et le bien-être de la population dans une perspective d'investissement pour la société.

Dans cet esprit, outre les dix-neuf objectifs et les six stratégies pour les atteindre, la politique insiste particulièrement sur quelques voies d'action indispensables à l'atteinte des objectifs qu'elle s'est fixés. II s'agit de :

- l'accentuation des activités de promotion/prévention;

- la concertation et le partenariat entre les établissements;

- l'intersectorialité.

Une lecture attentive et minutieuse de la politique nous a permis de relever près de cent cinquante passages où l'on fait référence d'une quelconque manière à l'une ou l'autre de ces trois voies d'action. L'importance que revêtent ces dernières vient non seulement du nombre de mentions dont elles font l'objet, mais également de leurs lieux et contextes d'apparition. En effet, ces mentions ne se confinent pas à la section portant sur les objectifs de santé et de bien-être : elles se répartissent à travers l'ensemble du document et sont introduites dès les premières pages.

Encore une fois, les Priorités nationales de santé publique 1997-2002 viennent renforcer l'accent mis sur ces voies d'action. Cela s'exprime, d'une part, par leurs buts qui consistent, entre autres, à promouvoir la santé et le bien-être de la population et à prévenir des problèmes de santé et des problèmes sociaux (p. 13); d'autre part, par l'adoption de principes directeurs, dont agir en amont des problèmes (Agir et comprendre, p. 25) et intervenir de façon concertée et coordonnée (p. 28).

La mise en place ou le renforcement de ces éléments fondamentaux sont sans contredit intimement associés à une performance optimale visée dans l'application de la PSBE. Ces voies d'action se présentent 
donc comme des variables intermédiaires devant absolument s'actualiser pour permettre l'atteinte des objectifs de la politique.

La mesure de ces résultats intermédiaires étant apte à rendre compte de la justesse de la démarche d'implantation de la politique, nous faisons de ces voies d'action les objets centraux de l'évaluation. Pour des raisons de lisibilité, nous ne parlerons dorénavant que de deux voies d'action, promotion/prévention et partenariat/concertation, la seconde incluant le volet intersectoriel.

\section{Le modèle d'évaluation}

Une fois les objets d'évaluation cernés, il nous fallait articuler l'évaluation autour d'eux, donc définir un modèle. Auger (1994) identifie neuf types d'évaluation distincts mais non mutuellement exclusifs dans leur application. Un même programme peut en effet profiter d'un ou de plusieurs types d'évaluation en fonction de son état de développement et de la nature des informations nécessaires à la prise de décision.

Ainsi, lorsque la démarche d'évaluation s'insère en cours d'action et qu'il s'agit d'un programme ou d'une politique non éprouvée, comme c'est le cas pour la PSBE, quatre types d'évaluation sont particulièrement indiqués : il s'agit de l'évaluation 1) d'implantation, 2) de structure, 3) de processus, et 4) de fonctionnement. Tenant compte des préoccupations formulées lors des diverses consultations, nous avons choisi de retenir les trois premiers types tout en les adaptant aux besoins d'information exprimés ainsi qu'aux contraintes situationnelles. Le quatrième type a quant à lui été intégré à l'évaluation de processus étant donné la grande parenté qui existe, tant dans les écrits que dans les faits, entre ces deux types d'évaluation.

\section{Les composantes du modèle}

\section{L'évaluation de structure}

D'après Thomas (1995), l'évaluation de la structure correspond à l'appréciation de la quantité, de la qualité et de l'organisation des ressources consacrées à un programme. Dans le cas qui nous occupe, les ressources qui sont consacrées à l'application des voies d'action proposées par la PSBE sont de deux ordres. Premièrement, il y a les documents de planification, qui informent nos partenaires des grandes 
orientations que privilégie la Régie régionale et de ses préceptes en matière de planification et d'organisation des services. II s'agit, par exemple, du Plan d'action triennal de la Montérégie 1994-1997 sur la Politique de la santé et du bien-être, du Programme régional de santé publique et du Plan régional d'organisation des services pour les personnes âgées.

Deuxièmement, on retrouve, en termes de ressources, les moyens dont s'est dotée la Régie, ou qui gravitent autour d'elle, et qui facilitent l'appropriation de ces documents. Il s'agit soit de mécanismes de concertation, tels les tables de coordination sous-régionale, comités programme clientèle, tables de concertation, etc., soit d'acteurs solo comme les conseillers sous-régionaux.

L'évaluation de ces deux types de ressources s'est faite séparément. En ce qui a trait aux documents, l'évaluation de la structure s'est attardée à en décrire les caractéristiques, l'agencement et l'adhésion aux principes préconisés par la politique.

\section{Tableau I}

Principales composantes du modèle d'évaluation

\begin{tabular}{cccc}
\hline $\begin{array}{c}\text { Types } \\
\text { d'évaluation }\end{array}$ & Objets & Intrants & Finalités \\
\hline Structure & Voies d'action PSBE & Ressources $^{2}$ & $\begin{array}{c}\text { Appréciation de la } \\
\text { contribution théorique }\end{array}$ \\
Implantation & $\begin{array}{c}\text { Voies d'action } \\
\text { PSBE }^{3}\end{array}$ & $\begin{array}{c}\text { Activités de } \\
\text { promotion/prévention } \\
\text { ou activités menées } \\
\text { en partenariat } \\
\text { ou en concertation } \\
\text { Processus }\end{array}$ & $\begin{array}{c}\text { Appréciation } \\
\text { de l'actualisation } \\
\text { des voies d'action }\end{array}$ \\
\hline Rossources & $\begin{array}{c}\text { Appréciation de la } \\
\text { contribution réelle }\end{array}$ \\
\hline
\end{tabular}

Du côté des mécanismes, l'évaluation de la structure a mené à la description de leurs principales caractéristiques, de leur composition, de l'organisation administrative entre les instances ainsi qu'à l'intérieur de celles-ci.

2. Documents de planification et mécanismes de participation.

3. Promotion/prévention et Partenariat/concertation (incluant Intersectorialité). 


\section{L'évaluation d'implantation}

Une évaluation d'implantation requiert qu'une comparaison soit effectuée entre, d'une part, des activités planifiées et, d'autre part, celles effectivement réalisées. Or, l'implantation des voies d'action retenues comme objets de l'évaluation ne figure à aucun plan d'action. II nous était donc impossible de soumettre ces voies d'action à une évaluation d'implantation, ou même à un suivi d'implantation en bonne et due forme. Nous avons par conséquent opté pour dresser un portrait évolutif de l'état d'implantation des voies d'action, ce portrait devant permettre de jauger dans quelle mesure les organisations empruntent les voies indiquées par la PSBE dans la poursuite de ses objectifs.

Ne perdant pas de vue que la démarche évaluative s'inscrivait dans une finalité formative, nous avons également cherché à recueillir, par l'évaluation d'implantation, des informations qui permettraient de trouver les correctifs à apporter dans l'éventualité où l'actualisation des voies d'actions nécessiterait un quelconque redressement. Pour ce faire, nous avons enrichi le portrait, d'une part, par une vérification de la présence de conditions facilitantes associées à chacune des voies d'action et, d'autre part, par une documentation des facteurs contextuels qui ont favorisé ou compromis leur actualisation.

\section{L'évaluation de processus}

L'évaluation de processus sert quant à elle à nuancer les informations issues de l'évaluation d'implantation et à interpréter avec plus de justesse les résultats produits par une intervention. Le principal intérêt qu'elle présente se situe dans les liens explicatifs et dynamiques qu'elle permet de tisser entre les différents constats issus de l'évaluation.

Alors qu'un courant de pensée associe très étroitement l'évaluation de processus à l'évaluation d'implantation par un questionnement de la clientèle rejointe et de la mise en place des services (Horne, 1995; McKenzie et Jurs, 1993), un second courant s'interroge sur la dynamique interne du programme (Samson-Saulnier, 1995). C'est pour cette seconde voie que nous avons opté. Dans cette optique, s'intéresser au processus équivaut à se demander si l'on fait les bonnes choses et si on les fait bien.

Appliqué à la PSBE, ce type d'évaluation s'attarde lui aussi aux ressources mises à contribution dans l'application de la politique, et ce, 
dans le but d'estimer leur contribution réelle à l'implantation des voies d'action retenues. Pour ce faire, l'évaluation de processus s'est attardée à trois volets distincts des documents et mécanismes introduits par la Régie régionale : 1) leur degré de notoriété et d'utilisation, 2) leur niveau d'adéquation étant donné les contraintes imposées aux divers acteurs du réseau et, enfin, 3) leur apport dans l'application des voies d'action.

\section{LES CHOIX RELATIFS À LA VALIDITÉ DE LA DÉMARCHE D'ÉVALUATION}

\section{L'unité d'observation : le programme clientèle}

L'unité d'observation correspond à l'univers à l'intérieur duquel se réalise l'évaluation. II doit être clairement circonscrit, car il détermine largement le choix de la méthode de collecte d'information à utiliser aux fins de l'évaluation. Étant donné les efforts consacrés, au moment d'entreprendre l'étude, par le MSSS, la Régie régionale ainsi que ses partenaires à l'organisation du travail et des données selon une approche par programme clientèle, nous avons fait de ce programme notre unité d'observation.

Tous les programmes clientèle n'ont cependant pu être inclus dans l'étude, notamment en raison du modèle d'évaluation privilégié et des ressources disponibles pour l'appliquer. II nous a donc fallu trouver une méthode qui nous permette d'opérer un choix des programmes clientèle qui soit le plus valide possible, tant du point de vue de leur représentativité que de la généralisation des résultats de l'évaluation.

Étant donné l'absence de critères absolus susceptibles de conduire à la sélection des programmes clientèle, nous avons eu recours à la technique du groupe nominal pour établir les critères à considérer. Ainsi, après une fructueuse séance de travail à laquelle participaient une dizaine de nos collègues, nous avons été en mesure de dégager cinq critères sur lesquels baser nos décisions. Ceux-ci ont guidé nos choix vers les programmes qui au moment de l'évaluation : 1) faisaient appel à plus d'une catégorie de fournisseurs de services, 2) faisaient l'objet d'ajouts de fonds, 3) entraînaient les dépenses les plus importantes, 4) étaient implantés dans l'ensemble de la région et, enfin, 5) étaient les plus visés par les objectifs de la PSBE.

En plus de ces critères, nous avons pris en considération les priorités nationales de santé publique, puisqu'elles sont le fruit d'une importante réflexion du réseau de la santé publique à l'échelle provinciale. 
Trois programmes clientèle se sont alors distingués. Deux d'entre eux, les programmes clientèle jeunesse et personnes âgées, ont finalement été retenus, principalement parce que leurs contenus ne se chevauchent que très peu. Cette distinction entre les deux programmes touchés par l'évaluation est avantageuse sur le plan méthodologique : elle améliore la validité externe de l'étude en augmentant le potentiel de généralisation des résultats de l'évaluation.

\section{Les milieux d'investigation}

En Montérégie, l'administration des services de santé et des services sociaux s'opérationnalise en passant par les sous-régions, qui sont au nombre de sept. Nous avons donc cherché à respecter ce découpage territorial au moment d'identifier les milieux au sein desquels puiser l'information nécessaire à nos travaux. Étant donné l'impossibilité, pour une question de disponibilité de ressources, de couvrir l'ensemble des sous-régions qui composent le territoire montérégien, nous avons dû effectuer une sélection parmi celles-ci.

Pour ce faire, nous nous sommes inspirés des travaux de Pampalon et collab. (1995). Ces auteurs proposent quatre types de zones pour décrire le degré d'urbanisation d'un territoire donné. Trois de ceux-ci s'appliquent à la Montérégie, soit les types métropolitain, agglomérations et villes ainsi que rural. C'est sur la base de cette catégorisation que nous avons effectué la sélection des sous-régions.

Dans un souci de représentativité, nous avons cherché à choisir des sous-régions qui, réunies, reflètent les trois types de zones. Comme le territoire de chacune des sous-régions comporte plus d'un type de zone, nous avons identifié, pour chaque type, la sous-région dont la proportion des ménages est la plus élevée, et nous l'avons retenue. II en résulte donc la sélection de trois sous-régions qui se veulent représentatives du territoire montérégien. Par ailleurs, le poids démographique étant fort variable d'une sous-région à une autre, nous avons veillé, lors de la composition de la banque de répondants, à respecter le plus possible cette réalité. Ainsi, dans notre étude, la sous-région la plus populeuse comporte un nombre plus important de répondants, et ce, bien qu'on ne s'intéresse d'aucune façon à comparer les sousrégions entre elles. 


\section{QUELQUES RÉSULTATS}

\section{L'évaluation de la structure}

Dans l'ensemble, les documents de planification qui furent élaborés dans la lignée de la Politique de la santé et du bien-être sont en accord avec elle. Ainsi, du point de vue de la planification, ils se situent tous, pour reprendre les expressions de Pineault et Daveluy (1995), à des niveaux inférieurs à celui où se trouve la politique (stratégique).

Par contre, une des caractéristiques de la planification, c'est l'action (Pineault et Daveluy, 1995). Tout plan devrait donc déboucher sur une programmation opérationnelle. Or, l'évaluation aura permis de constater que la plupart des outils analysés ont du mal à se distancier du niveau stratégique de planification. Sachant que l'absence ou la déficience d'un des niveaux (stratégique, tactique, opérationnel) affaiblit tout le système de planification, il y a lieu de s'inquiéter en présence d'outils dont le degré de précision ou de hiérarchisation des priorités est insuffisant compte tenu du rôle qu'on attend d'eux.

En ce qui a trait à la valorisation des voies d'action proposées par la PSBE, tous les outils de planification analysés font une place importante à la promotion/prévention, en ce sens qu'ils en contiennent de très nombreuses mentions. Cependant, une analyse plus fine a permis de constater que cette importance est quelquefois plus apparente qu'effective.

Du point de vue théorique, on constate par ailleurs que les mécanismes de concertation étudiés respectent en grande partie les voies d'action préconisées par la PSBE, notamment en matière de mandat et de composition. Les questions de partenariat/concertation sont véritablement à l'avant-scène, mais aucune définition de ces voies d'action n'est offerte, tout comme dans les outils de planification. En outre, on ne trouve ni modèle de concertation, ni proposition de façon de faire pour assurer le développement du partenariat, quelle que soit la forme qu'il prenne. Par conséquent, le passage de la théorie à la pratique risque de se heurter à des obstacles de taille.

\section{L'évaluation d'implantation}

En ce qui a trait à l'évolution de la proportion des activités de promotion/prévention, c'est plus de la moitié des répondants qui nous ont 
informé d'un accroissement au cours des dernières années. Toutefois, la tendance rapportée est nettement plus prononcée chez les acteurs du secteur communautaire et bénévole que chez ceux de la santé et des services sociaux.

Aussi, chez une proportion non négligeable d'acteurs, tant du réseau de la santé que de l'extérieur du réseau, la situation est stagnante depuis cinq ans, voire en régression. Qui plus est, le pronostic de ces répondants pour les années à venir est encore moins réjouissant. Dans l'un et l'autre cas, cette situation s'explique en bonne partie par les restrictions budgétaires effectuées au cours des dernières années et qui ont affecté l'ensemble de nos partenaires du réseau, y compris, dans le cas de la Montérégie, les centres locaux de services communautaires (CLSC). Lorsque la pression se fait sentir sur les soins et services fournis par ces derniers, il semble donc qu'elle soit dirigée en priorité sur la promotion et la prévention.

D'après plus d'une centaine d'acteurs, le pronostic en matière de partenariat est généralement encourageant. Quand on examine l'état actuel du partenariat ainsi que son évolution au cours des cinq dernières années, force est de constater qu'il semble assez bien se porter. Cette constatation vaut surtout pour les organismes communautaires mais s'applique également, quoiqu'à un degré moindre, aux établissements du réseau. Par contre, les partenaires hors réseau font quelque peu bande à part, le partenariat faisant beaucoup moins partie de leurs pratiques.

Quand on se tourne vers l'avenir, il y a des raisons d'être optimistes, puisque la majorité des répondants prévoient une augmentation des activités de partenariat. Encore une fois, ce sont les acteurs hors réseau qui se montrent plus hésitants à cet égard. Finalement, un travail important reste encore à faire auprès de ces derniers si l'on souhaite les amener à travailler davantage en partenariat avec les acteurs du secteur de la santé et ceux des milieux communautaires.

En ce qui concerne la concertation, les résultats indiquent qu'elle est plus difficile à réaliser surtout lorsqu'elle est imposée de l'extérieur. C'est d'ailleurs le cas des tables de coordination sous-régionales. La concertation constitue une entreprise de longue haleine qui nécessite la mise en place d'un nombre important de conditions de réussite. Par contre, lorsqu'elle vient du milieu, comme c'est le cas pour certains comités ou groupes de travail, la concertation se porte mieux. 


\section{L'évaluation du processus}

L'évaluation du processus visait, entre autres choses, à documenter la contribution de la RRSSSM en matière de promotion/prévention et de partenariat/concertation. Elle aura permis de mieux comprendre pourquoi il existe une difficulté à transcrire les visées de la Régie régionale en actions concrètes. Ainsi, une partie de la réponse renvoie à des conditions exogènes à la Régie régionale sur lesquelles cette dernière n'a pas de véritable emprise. On parle ici notamment de restrictions budgétaires, de manque de ressources, de choc des cultures.

Par contre, ces facteurs exogènes ne peuvent seuls être tenus responsables de la faible contribution de la Régie régionale à l'implantation des voies d'action de la PSBE. En effet, l'évaluation de processus a mis en évidence que la notoriété et l'utilisation, tant des outils de planification développés à la Régie régionale que des mécanismes de concertation, sont insuffisantes. La faible utilisation serait attribuable au fait que les outils de planification correspondent très imparfaitement à la réalité des acteurs sur le terrain, soit par leur contenu peu réaliste, leur formulation peu opérationnelle ou leur présentation volumineuse.

Certains constats issus de l'évaluation du processus confirment que tous les efforts de la Régie régionale n'ont cependant pas été vains et qu'un engagement plus ferme de sa part serait même bienvenu. En effet, nos partenaires disent apprécier l'aspect informationnel des outils de planification et la gouverne qu'ils fournissent. Qui plus est, au chapitre des attentes exprimées par les informateurs clés, figurent plusieurs mentions faisant état du rôle de chef de file attendu de la Régie régionale. Bref, loin de souhaiter que la Régie régionale se tienne sur les " lignes de côté ", on souhaite et on s'attend à ce que celle-ci soit proactive auprès de ses partenaires.

\section{CONCLUSION}

La démarche évaluative que nous avons réalisée, et qui a fait appel à une approche novatrice en matière d'évaluation de politiques, a prouvé qu'il est possible, malgré des ressources humaines et financières relativement modestes, de réaliser une évaluation formative d'une politique d'envergure nationale. De plus, les choix conceptuels que nous avons effectués ont permis à l'évaluation de demeurer au-dessus de la mêlée en se distanciant des programmes à travers lesquels 
s'actualise la PSBE. Cette façon de faire nous a ainsi permis de concentrer nos efforts sur un nombre limité d'objets et, ce faisant, de jauger, de manière macroscopique, le degré d'implantation globale de la politique dans notre milieu.

Le bilan de l'application de notre démarche évaluative permet de croire que ces choix furent heureux, tant du point de vue conceptuel que méthodologique. En effet, cette démarche nous a permis de recueillir une masse d'informations à la fois nuancées et convergentes à travers les trois types d'évaluation employés.

La démarche d'évaluation présente cependant certaines limites qui ont essentiellement trait à la validité externe des résultats auxquels elle a conduit. Ainsi, le moment venu de sélectionner les programmes clientèle, il nous a fallu effectuer des choix à partir de critères qui avaient peu à faire avec le principe d'une éventuelle généralisation des conclusions de l'étude. Bien que la notion de généralisation fût partiellement prise en considération dans le choix final des programmes clientèle, on ne peut pour autant affirmer sans équivoque que la sélection d'autres programmes nous aurait conduits à des résultats en tous points identiques à ceux obtenus.

Même si notre sélection des milieux d'investigation repose sur un souci de représentativité, il n'en demeure pas moins que notre stratégie de sélection, basée sur les travaux de Pampalon et collab. (1995), n'a peut-être pas permis de refléter fidèlement l'importance réelle de chacun des trois types de zones dans la composition du territoire montérégien. Par conséquent, il se peut que les sous-régions sélectionnées ne rendent pas tout à fait justice à l'ensemble de la région, d'autant plus qu'elles font preuve d'idiosyncrasies en matière d'organisation des services, de partenariat et de concertation.

La démarche que nous avons élaborée dans le but d'effectuer une évaluation formative de la PSBE est centrée sur les voies d'action qu'elle propose et non sur des programmes spécifiques par lesquels elle s'actualise. Tant l'approche élaborée que les instruments développés peuvent donc être repris par d'autres régies régionales intéressées à connaître le degré d'actualisation des voies d'action dans leur milieu, puisque toutes procèdent suivant un découpage plus ou moins fin de leur territoire en plus de traiter avec huit programmes clientèle. 
Pour les régies régionales qui desservent de petits territoires, ou des territoires dont le degré d'urbanisation est relativement uniforme, notre stratégie de sélection des milieux d'investigation sera cependant de peu de secours. Par ailleurs, rares sont les régies régionales qui ont retenu comme cibles d'action les dix-neuf objectifs de santé que l'on retrouve dans la Politique de la santé et du bien-être. Dans les organisations où l'on a procédé à une sélection, il va sans dire que le choix des programmes clientèle devra se faire à la lumière des priorités déjà fixées afin de donner préséance aux programmes qui sont en lien direct avec les objectifs retenus.

Malgré les quelques limites méthodologiques mentionnées et la nécessité pour les régies d'adapter la stratégie de sélection des unités d'observation et des milieux d'investigation à leur propre réalité, il n'y a pas lieu pour autant de freiner l'emprunt de l'approche que nous avons élaborée. Celle-ci s'est en effet avérée fructueuse, puisque les résultats obtenus s'accordent avec ceux d'une étude réalisée en Montérégie sur la concertation au sein des tables sous-régionales ainsi qu'avec les résultats de deux consultations menées depuis 1996 auprès des partenaires de la Régie régionale. II y a donc tout lieu de croire que la démarche empruntée a permis une lecture assez fidèle de la réalité associée à l'actualisation des voies d'action de la politique sur le territoire montérégien.

Sylvie CANTIN Agente de planification, programmation et recherche Direction de la santé publique, de la planification et de l'évaluation

Régie régionale de la santé et des services sociaux de la Montérégie

Jean BEAUDRY Agent de recherche sociosanitaire

Direction de la santé publique, de la planification et de l'évaluation

Régie régionale de la santé et des services sociaux de la Montérégie 


\section{Références bibliographiques}

AUGER, G. (1994). Cadre de référence pour l'évaluation des interventions en santé au travail, RRSSS de Lanaudière, Direction de santé publique.

BÉLAND, François (1983). L'Évaluation de la politique des services à domicile du ministère des Affaires sociales : étude de cas, Service évaluationsanté, Direction de l'évaluation des programmes, ministère des Affaires sociales.

BOLDUC, Mario (1986). "Les clientèles ou problématiques considérées ", Analyse de la cohérence de politiques québécoises à l'égard des personnes ayant des incapacités, no 24 , Direction générale de l'évaluation et de la planification, ministère de la Santé et des Services sociaux.

HORNE, T. (1995). Making a Difference : Program Evaluation for Health Promotion, Well Quest Consulting Ltd. (ed.).

MCKENZIE, J.F. et J.L. JURS (1993). Planning, Implementing and Evaluating Health Programs : A Primer, McMillon Publishing Company.

MINISTÈrR DE LA SANTÉ ET DES SERVICES SOCIAUX (1997). Priorités nationales de santé publique 1997-2002, Gouvernement du Québec.

Ministère de LA SANTÉ ET DES SERVICES Sociaux (1995). Les fiches descriptives des indicateurs de la politique québécoise de la santé et du bien-être, Direction générale de la planification et de l'évaluation. (Méthodologie et instrumentation, no 9)

Ministère de LA SANTÉ ET DES SeRvices sociaux (1992). Politique de la santé et du bien-être, Gouvernement du Québec.

MINISTĖRE DES AFFAIRES SOCIALES (1997). Une politique québécoise en matière de nutrition, Direction générale de la planification, Service des études épidémiologiques.

PAMPALON, R. et collab. (1995). Variations géographiques de la santé, Rapport de l'Enquête sociale et de santé 1992-1993, vol. 3, Montréal, Santé Québec, ministère de la Santé et des Services sociaux.

Pineault, R. et C. Daveluy (1995). La planification de la santé (2e éd.), Montréal, Éditions Nouvelles.

SAMSON-SAULNIER, G. (1995). L'évaluation de programme : une démarche intégrée dans les établissements de santé et de services sociaux, Montréal, CLSC Villeray.

STECHER, Brian M. et W. Allan DAVIS (1987). How to Focus an Evaluation, Newbury Park, Sage.

THOMAS, D. (1995). Guide pour l'évaluation des interventions en prévention et en promotion de la santé et du bien-être, RRSSS de Lanaudière, Direction de la santé publique. 